\section{RECENT WORK AT HARVARD COLLEGE OBSERVATORY.}

THE forty-ninth annual report of the Director of the Harvari College Astronomical Observatory, by Prof. E. C. Pickering, has come to hand. Omitting matters of adininistration and some of the details, the following summary shows the most important events of the year covered by the report.

\section{Photometric Observations.}

The reduction of the photometric measures of the southern stars observed by Prof. S. I. Bailey in Pera, is now completed, and the catalogue containing the resulting magnitudes is in print. The observations of the first investigation undertaken with this instrument since its return from Peru are nearly completed. About six thousand stars have each been observed on at least three evenings. This catalogue includes all the stars of the Harvard Photometry, eighty lists of comparison stars for variables of long periods, and various stars the magnitudes of which are desired by other astronomers. A study has been made of the atmospheric absorption, especially for very low stars, and its coefficient has been derived on each evening, both for the southern and the northern stars. These values are now applied as corrections to the individual observations, instead of adopting a mean value of the absorption. A new working list has been prepared of the stars north of $-40^{\circ}$ having magnitudes 7.5 or brighter, and which have not already been observed with the meridian photometer. This list contains about fourteen thousand stars, of which about two thousand have been observed during the last year.

Prof. Pickering refers to Mr. S. C. Chandler's criticisms on the photometric observations made with the meridian photo. meter. (Astron. Nach. vol.cxxxiv. p. 355, and vol. cxxxvi. p. 85.) It is maintained that the meridian photometer possesses the same advantages in measuring the light of a star that the meridian circle does in measuring its position. In both instru. ments absolute values are determined directly, and they are obtained very rapidly. Stars are identified in the same way in both, rapidly and accurately, and in both the systematic errors are small, even if the accidental errors are in some cases larger than those resulting from other methods. Stars can be observed with the meridian photometer under favourable cir. cumstances nearly at the rate of one a minute, and the average deviation of the results thus obtained does not generally exceed one-tenth of a magnitude.

\section{Astronomical Photography.}

The number of photographs taken with the 8 -inch Draper telesco,se is 1657 . The number taken in Peru with the 8 inch Bache telescope is 1708 . All of the spectra photographed with these instruments have been examined by Mrs. Fleming. As a result seven variable stars, U Pappis, V Cancri, V Leonis, T Sagittarii, R Delphini, R Vulpeculæ, and R Phcenicis, have been shown to have the hydrogen lines bright in their photographic spectra. Unsuccessful attempts have been made to photograph the spectra of many other variables of long period when at maximum, and no image has been obtained. This has been found to be due in many cases to large errors in the ephemerides, and has been remedied by depending upon the observations of Mr. W. M. Reed, and making the time of photographing each star depeud upon its observed, instead of its predicted, brightness. Eleven new variables have błen discovered in the year, from the presence of bright hydrogen lines in their spectra, besides three the variability of which was discovered from changes in their photographic images. The number of stars of the fifth type has been incieased by seven, making the total number of these objects 62 . Five nebulæ have been discovered from their spectra. The hydrogen line $\mathrm{H} \beta$ has heen discovered to be hright in the spectra of five stars, and twelve stars have been shown to belong to the fourth type. The spectra of A, G. C. 18049 and 22640 are peculiar. Several photographs have been obtained of the new star in the constellation Norma. At the Lick Observatory it was shown from visual observations that its, spectrum, as in the case of the new star in Auriga, had become that of a gaseous nebula. This has been confirmed from photographs talien at Arequipa, which also show that this object is now gradually becoming fainter. The spectra of 4557 stars on thirty plates have been classified for the new Draper Catalogue. On one of these plates covering the region of the variable star $\eta$ Carinæe, $116 \mathrm{I}$ spectra have been measured.

The number of photographs taken with the II-inch Draper telescope is 912 . The lines in the spectrum of $\zeta$ Ursæ Majoris are found to be double in 59 out of $34^{\circ}$ images, and of $\beta$ Aurigae in 47 out of 65 .

An investigation has been in progress for some time for the detection of stars having large parallaxes or proper motions. Photographs are taken in the usual position with the film towards the object glass, and also with the plate reversed, the photograph being taken through the glass. These are repeated at intervals of six months at the times when the effect of parallax on their right ascension would have its greatest value. Plates thus obtained may then be superposed so that th= filins shall be in contact, and the two images of each star made to appear like a close double with components north and south. Sinall changes in position may be detected by changes in the position angle, and the amount of the parallax or proper motion may be measured with great accuracy. Several hundred stars have thus been shown to have no parallax exceeding half a second.

\section{Observation in PrRu.}

The meteorological station on the summit of Misti, at a height of 19,200 feet, was succesifully conduc'ed for several months, one of the assistants, generally Mr. Waterbury, visiting it every ten days, and readjusting the self-recoriling instru. ments. Unfortunately, early in September, the shelter containing the instrument; was found to have been broken into, and a number of the instruments carried off. Apparently the robbery was committed by two Indians, whose tracks were followed to a considerable distance. The property stolen would, of course, be of no uve to the thieves, and its intrinsic value would be a small part of the actual loss. The work at this station was conducted with great labour; a mule path had been built to the summit, and the entire expenditure had been large. It will be a serious loss to science if it proves impossible to maintain the station.

The number of photographs obtained by Prof. Bailey with the 13 -inch Boyden telescope is $56 \mathrm{I}$, including some remarkable charts. Among them may be mentioned photographs of the Nebula of Orion with an exposure of eight hours, of the cluster $\omega$ Centauri with an exposure of six hours, and of $\eta$ Carinæ with exposures of six and fourteen hours respectively. In some of these, notwithstanding the long exposure, the image shows no deviation from the circular form. This is mainly due to substituting for a finder two eyepieces attached to the main telescope. One of them serves to follow a guiding star in the usual way; the other, directed to another star, shows if the plate needs to be rotated in its own plane. This appears to be an important improvement in making the best photographic charts of long exposure, especially of the polar regioss. By the ordinary methods it is impossible to entirely correct such errors as those due to flexure and refraction, which do not depend upon the direction of the axis of the earth.

Attention is again drawn to the importance of making use of the admirable atmospheric conditions at Arequipa. A telescope of the largest size would not only have most favourable opportunities for work, but a field unexplored with such an instrument in the southern sky. Much could be done with a smaller instrument, as is shown by the work already accomplished with the 13-inch Boyden telescope. Out of a list of thirty telescopes having aperture exceeding 14 inches or more, but one is mounted south of latitude $+35^{\circ}$, and this one is not in use. A moderate expense only would be required to carry out this plan.

\section{WORK WITH THE NEW Telescope.}

One of the most important events of the year was a careful trial of the Bruce photographic telescope. Nearly a thousand photographs have been obtained with it. The spectra of the faint stars prove very satisfactory, and stars too faint to be photographed with the other instruments can thus be studied. Bright hydrogen lines have been found in the spectra of S Orionis, $S$ Bootis, W Virginis and $S$ Libræ, and the spectrum of $V$ Ophiuchi has been shown to be of the fourth type. The length of these spectra is about a quarter of an inch, which is sufficient to show much detail in the spectra of fairly bright stars. To study still fainter spectra a prism of crown glass and of smaller angle has been ordered. The absorption of the photographic rays is less for this material, and it is expected that much fainter 
spectra can be photographed, since the dispersion will also be less. The contract has not yet been filled for the Bruce telescope, since difficulty is still experienced in making charts in which the images shall be circular. Experiments are in progress in this direction, and it is hoped that the method described above as applied to the 13 -inch Boyden telescope will prove equally successful with this instrument.

A variety of experiments have been made to determine the photographic magnitudes of the brighter stars on a unifurm scale. It is now expected that this can be done with the transit photometer for stars brighter than the third magnitude, and that the scale can be extended to stars from the third to the sixth magnitude by a series of photographs which are being taken with a portrait lens having an aperture of 2.5 inches. The images are thrown out of focus, and the intensity of the circular discs thus obtained can be accurately measured.

\section{ELECTRIC DISCHARGE THROUGH GASES. ${ }^{1}$}

$\mathrm{ONE}$ of the most important and interesting branches of physical science is that which deals with the connection between electrical and chemical effects.

The investigations on electrolysis made within these walls by Davy and Faraday proved that the important class of electrical phenomena associated with the passage of electricity through liquids, are connected in the closest way with chemical action. They proved that no electricity will pass through most liquids unless chemical action occurs, and that for each unit of electricity which passes through the liquid there is a definite amount of chemical decomposition.

This care, though it is one where the laws are most accurately known, is but one among many electrical phenomena which are inseparable from chemical action.

So many instances of this kind have been discovered, that we may perhaps venture to hope that we are not far from the time when it will be universally recognised that many of the most fundamental questions in chemistry and electricity are but different aspects of one and the same phenumenon.

Anything which throws light on the connection between electricity and matter, interesting as it is on its own account, acquires additional interest when regarded as elucidating the connection between chemical and electrical effects, and no phenomena seem more suitable for this purpose than those which are the subject of the discourse this evening-the discharge of electricity through gases. For in gases we have matter in the state in which its properties have been most carefully studied, while the investigation of the electrical effects is facilitated by the visibility of the discharge, affording us ocular, and not merely circumstantial, evidence of what is taking place.

The points to which I wish to refer particularly this evening are, firstly, some phenomena connected with the passage of electricity from the gas to the electrode, or from the electrode to the gas; and secondly, some of the properties of the discharge when its course lies entirely in the gas.

By taking a long discharge tube, say, one fifty feet long, and observing the luminous discharge through a rotating mirror, we can trace the course of the luminosity due to a single discharge, say, one due to once breaking the primary circuit of an induction cuil ; if we do so, we find that the luminosity follows the direction of the positive current through the tube. That is, the luminosity begins at the positive electrode, it then rushes down the tube with enormous velocity, but when it gets to the negative electrode, it receives a check; it does not disappear at once in that electrode like a rabbit going down a hole, but lingers around the electrode some time belore entering it. In consequence of this delay in the positive discharge in getting out of the gas, there is an accumulation of positive electricity in the neighbourhood of the negative electrode until the potential fall at this electrode increases to about 200 or 300 volts.

The positive electricity which accompanies the discharge thus finds considerable difficulty in getting from the gas to the metal, though, as I hope to show you later on, as long as it keeps in the gas, it meets with what we may, in consideration of the views sometimes enunciated on this subject, call a ridiculously small amount of resistance, its real difficulty is to get out of the gas.

Though this effect has long been known, it is so important

1 Lecture delivered at the Royal Institution by Prof. J. J. Thomson, F.R.S. that I will venture to show one or two experiments which illustrate it. The arrangement of the first experiment is shown in Fig. I. The apparatus consists of a main discharge tube, across which is fastened a diaphragm made of excessively thin platinum leaf; there is a side passage from the tube, leading from one side of the diaphragm to the other, this is connected to a barometer tube, and by raising the cistern containing the

Fig. 1.

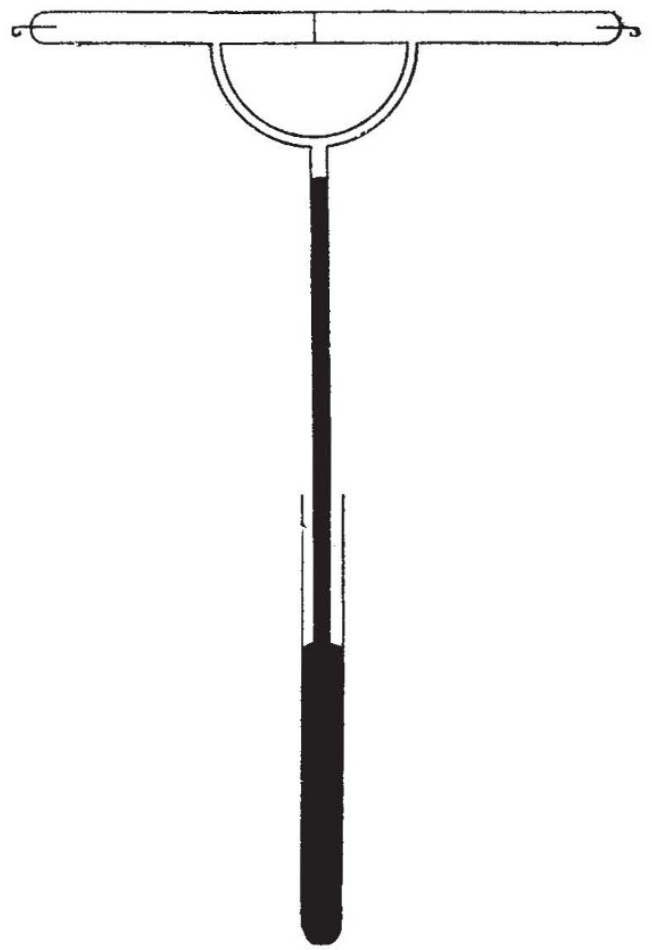

mercury I can stop up the passage by a pellet of mercury. We will first observe the discharge when the side passage is open; you see that the discharge, instead of passing across the thin piece of platinum leaf, takes the very much longer route round the side tube, so as to avoid crossing the metal. We will now raise the mercury cistern, and close the side tube by a pellet of mercury; the discharge now has no alternative but to cross the

Fig. 2 .

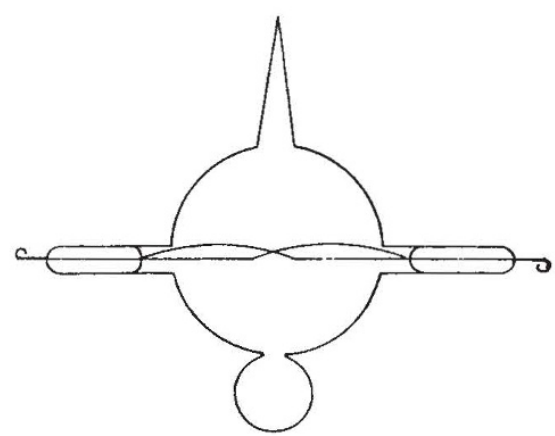

metal at some part of its course, and you see that the main portion of the discharge goes back into the main tube.

In the second experiment the metal diaphrag $m$ is replaced by a very thin plate of mica; when the side passage is opened the discharge goes round, but when this is closed by a pellet of mercury the discharge prefers to go across the mica than through the mercury.

$$
\text { No. } 13 \text { [8, voL. } 51]
$$

